\title{
Primary contraction of skin grafts: a porcine preliminary study
}

\author{
Alexander Bogdanov Berezovsky, Vasileios A. Pagkalos, Eldad Silberstein, \\ Yaron Shoham, Lior Rosenberg, Yuval Krieger
}

Department of Plastic and Reconstructive Surgery, Soroka University Medical Center, Ben Gurion University of the Negev, P.O. 151, Beer-Sheva 84101, Israel.

Address for correspondence: Dr. Vasileios A. Pagkalos, Division of Plastic and Reconstructive Surgery, Soroka University Medical Center, Ben-Gurion University of the Negev, P.O. Box 151, Beer-Sheva 84101, Israel. E-mail: pagkalos_v@yahoo.gr

\begin{abstract}
Aim: Skin grafting is a common clinical practice for plastic surgeons, yet primary contraction of these grafts is a neglected topic. This study was designed to investigate primary contraction and introduce the shape of skin graft as a possible factor that modifies primary contraction behavior, using porcine models. Methods: In the first series, full-thickness skin grafts (FTSGs) and split-thickness skin grafts (STSGs) were compared. In a second series, how the shape of the skin graft affected the degree of contraction was examined. Results: The mean percentage of FTSG shrinkage was $12.04 \%$, and the median was $12.18 \%$. The mean percentage of STSG shrinkage was $6.87 \%$, and the median was $5 \%$. Circle-shaped and square-shaped FTSGs showed mean/median graft shrinkage of $5.83 \% / 6.93 \%$ and $4.15 \% / 3.75 \%$, respectively. In STSGs, the circle-shaped and square-shaped grafts had mean/median graft shrinkage of $1.07 \% / 0 \%$ and $0.31 \% / 0 \%$, respectively. Conclusion: Our preliminary report revealed an expected greater shrinkage of FTSGs compared with STSGs. Furthermore, in a limited number of specimens, the shape of the skin graft seemed to affect the primary contraction of the STSGs.
\end{abstract}

Key words:

Animal model, full-thickness skin graft, primary skin contraction, split-thickness skin graft

\section{INTRODUCTION}

Wound contraction is a normal physiological phenomenon reducing the area of a skin defect and therefore expediting its closure. This contraction is based on scar contraction and myofibroblast activity; all originate from granulation tissue that develops during the 1 st week of the inflammatory process, part of the normal wound-healing course. The application of skin grafts to fresh skin defects has been proven to reduce wound contraction and hypertrophic scarring compared with full-thickness wounds that have been left to granulate and heal by secondary intention alone. ${ }^{[1,2]}$ However, skin grafts can also

\begin{tabular}{|l|l|}
\hline \multicolumn{2}{|c|}{ Access this article online } \\
\hline Quick Response Code: & Website: \\
\hline & www.parjournal.net \\
\cline { 2 - 3 } & \\
\hline & \\
\hline
\end{tabular}

contract, resulting in a compromised esthetic outcome and restricted mobility of the joints involved.

Skin graft contraction occurs in two stages: primary and secondary contraction. Primary contraction refers to the immediate reduction in size of the skin graft, directly after it has been harvested from its donor site. Primary contraction is due to passive recoil of the elastin fibers in the dermis and is, therefore, dependent upon the thickness of the graft. Full-thickness skin grafts (FTSGs) contain large volumes of elastin-containing dermis and consequently exhibit the greatest degree of primary contraction. Due to the reduced volume of dermis included, spilt-thickness skin grafts (STSGs) exhibit less contraction, whereas pure epidermal grafts do not contract. ${ }^{[3]}$ Secondary contraction is due to a wound bed contraction. This secondary contraction reduces both the size of the graft at the interface with its recipient bed and the circumference of the graft at its periphery. ${ }^{[1,4]}$ Traditionally, it is accepted that the degree of secondary contraction is inversely related to the thickness of the graft of FTSGs to minimize the extent of secondary contraction. ${ }^{[5]}$ Studies have shown that a granulating recipient bed, burn size, young age 
of the patient, anatomical area and grafting over mobile tissues may prompt skin graft contraction. ${ }^{[1,5-7]}$

Skin grafting is a major element of reconstructive surgery. It is, therefore, important that every aspect of its practice is thoroughly investigated and evaluated. Primary contraction was first described and assessed by Davis and Kitlowski in $1931 .{ }^{[3]}$ The authors of that pioneering study used human specimens, a practice that has some limitations regarding the number, size and shape of skin grafts. Furthermore, although $>80$ years have elapsed, there have not been any studies that further looked further into the development and cause of primary contraction. In this preliminary in vivo porcine study, we assessed the degree of primary skin graft contraction and investigated whether the shape of skin grafts affects the degree of contraction.

\section{METHODS}

The study was conducted at an accredited animal research facility (CRO, Lahav Research Institute, Lahav, Israel) following national and institutional guidelines for the care and use of laboratory animals. ${ }^{[8]}$ The animals were anesthetized female domestic pigs (Susscrofa), weighing approximately $30 \mathrm{~kg}$. The study was approved by the Institutional Animal Care and Use Committee.

The study consisted of two parts. In the first part, we compared the contraction of FTSGs and STSGs. Four animals participated in the study, and a total of 67 specimens of skin grafts were harvested from the back of the pigs, 41 and 26 of FTSGs and STSGs, respectively.

For the study of the FTSG contraction, circles of $4 \mathrm{~cm}$ diameter were drawn with a permanent marker on the skin of each pig [Figure 1]. The area of the circles marked was calculated using the formula: area $=\pi r^{2}$, where $r=$ radius of the circle and $\pi=3.14$, resulting in the area value of $12.56 \mathrm{~cm}^{2}$. The skin was excised with a No. 15-blade in a

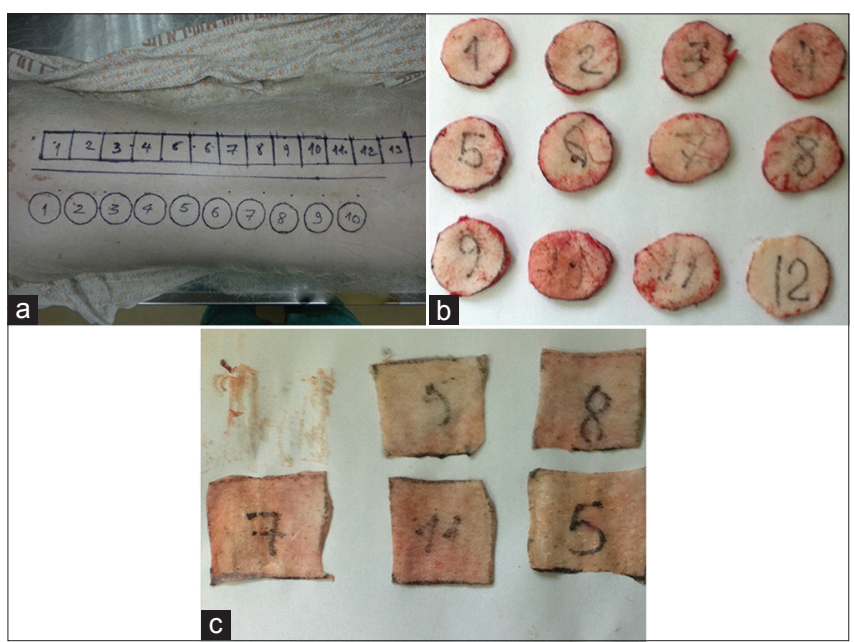

Figure 1: First part of study. (a) Skin grafts marked on the back of the animal. Circles are drawn with a permanent marker for the full-thickness skin grafts (FTSGs) and squares for the split-thickness skin grafts (STSGs). (b) Full-thickness skin grafts after excision and defattening. (c) STGSs after excision circle shape and meticulously defatted. The FTSGs were placed on a flat surface 15 min after skin graft harvesting to facilitate complete primary contraction; their diameter was measured in 3 axes (with an axis-to-axis angle of $120^{\circ}$ ) and the average diameter was used to calculate the radius of the circle [Figure 1]. The mean value of the diameter was computed, and the surface was again calculated using the formula: area $=\pi r^{2}$.

For the study of STSG contraction, a rectangle stripe of $4 \mathrm{~cm}$ height and $40 \mathrm{~cm}$ width was drawn with a permanent marker on the pig. Additional lines were drawn vertically to produce 10 squares of equal $4 \mathrm{~cm}$ sides [Figure 1]. The area of each square was calculated using the formula: area $=s^{2}$, where $s=$ side of the square and the initial area value of $16 \mathrm{~cm}^{2}$ were recorded. All STSGs were harvested with a dermatome adjusted to 0.014 inch skin thickness, corresponding to moderate to thick skin grafts. The stripe was cut into squares according to our drawings, and the grafts were placed on a flat surface and left there for $15 \mathrm{~min}$ before measuring [Figure 1]. Due to the primary contraction, the initial squares changed into rectangles. Each side of the contracted STSGs was, therefore, measured, and the surface was calculated using the formula: area $=h \times w$, where $h=$ height and $w=$ width

In the second part, we examined whether the shape of the skin graft affected the degree of contraction. For that purpose, we took a total of 27 specimens, harvested from the back of a single pig [Figure 2]. Nine of these specimens were circle-shaped FTSGs, 6 were square-shaped FTSGs, 8 circle-shaped STSGs and 4 square-shaped STSGs. The harvesting and calculation of the area of FTSGs and that of square-shaped STSGs were made in the same way as in the first stage of our study, with the initial surface area being $12.56 \mathrm{~cm}^{2}$ and $16 \mathrm{~cm}^{2}$ for the circle- and square-shaped specimens, respectively. For harvesting circle-shaped STSGs, we used a dermatome to create a "skin belt" graft, which was subsequently cut into a circle-shaped skin graft with scissors. All STSGs were harvested with a dermatome adjusted to 0.014 inch skin thickness [Figure 2].

Statistical analysis was performed using Microsoft Excel $2003^{\circledR}$ (Microsoft, Redmond, Washington, USA) and SPSS ${ }^{\circledR}$ version 14 (IBM-SPSS Inc., New York, USA). Statistical tests used Pearson's Chi-square test and Student's $t$-test.

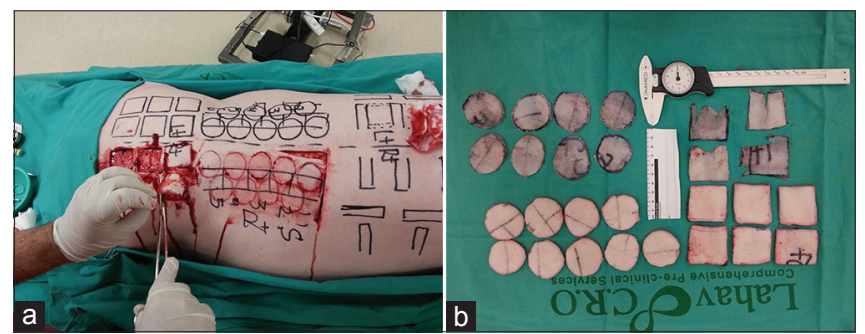

Figure 2: Second part of study. (a) Investigation of possible relation of shape of skin graft with degree of primary contraction. Circle- and square-shaped skin grafts excised from the back of a single animal. (b) Circle- and square-shaped full-thickness skin grafts and split-thickness skin grafts after excision 


\section{RESULTS}

In the first part of the study, comparison was made between the contractions of FTSGs and STSGs. The initial surface area of the FTSG specimens was $12.56 \mathrm{~cm}^{2}$. After excision and defatting, the area values of the contracted skin grafts ranged from $12.6 \mathrm{~cm}^{2}(0 \%$ shrinkage $)$ to $9.3 \mathrm{~cm}^{2}$ (25.6\% shrinkage). The mean area value of the FTSGs after primary contraction was $11.1 \mathrm{~cm}^{2}$ and the median was $11.0 \mathrm{~cm}^{2}$. The mean percentage of graft shrinkage was $12.0 \%$ and the median was $12.2 \%$ [Table 1]. The initial surface area of the STSG specimens was $16 \mathrm{~cm}^{2}$. After harvesting with the dermatome, the area values of the contracted skin grafts ranged from $16 \mathrm{~cm}^{2}(0 \%$ shrinkage, 1 specimen recorded) to $13.30 \mathrm{~cm}^{2}$ (17\% shrinkage). The mean area value of the STSGs after primary contraction was $14.9 \mathrm{~cm}^{2}$ and the median was $15.2 \mathrm{~cm}^{2}$. The mean percentage of graft shrinkage was $6.9 \%$ and the median was $5.0 \%$ [Table 1]. FTSGs presented greater primary contraction than STSGs at a statistically significant level $(P=0.0011)$ [Table 1].

In the second part, the role of the skin graft shape on primary skin contraction was investigated. The initial surface area of the circle-shaped specimens of both FTSGs and STSGs was $12.6 \mathrm{~cm}^{2}$ and the initial surface area of the square-shaped specimens was $16.0 \mathrm{~cm}^{2}$. After excision and defatting, the circle-shaped FTSGs had a calculated surface range from $12.3 \mathrm{~cm}^{2}$ (2.0\% shrinkage) to $11.3 \mathrm{~cm}^{2}$ ( $9.8 \%$ shrinkage). The mean area value of the circle-shaped FTSGs was $11.8 \mathrm{~cm}^{2}$ and the median was $11.7 \mathrm{~cm}^{2}$. In this group, the mean percentage of graft shrinkage was $5.8 \%$ and the median was $6.9 \%$ [Table 2]. On the other hand, the square-shaped FTSGs ranged from $15.6 \mathrm{~cm}^{2}(2.5 \%$ shrinkage $)$ to $14.8 \mathrm{~cm}^{2} \quad(7.4 \%$ shrinkage). The mean area value of the square-shaped FTSGs was $15.3 \mathrm{~cm}^{2}$ and the median was $15.4 \mathrm{~cm}^{2}$. In the square-shaped FTSGs group, the mean percentage of graft shrinkage was $4.2 \%$ and the median was $3.8 \%$ [Table 2].
The comparison of primary contraction values between square- and circle-shaped FTSG specimens was not statistically significant $(P=0.14)$. The circle-shaped STSG specimens demonstrated a primary contraction ranging from $12.6 \mathrm{~cm}^{2}$ ( $0 \%$ shrinkage) to $11.9 \mathrm{~cm}^{2}$ (5.0\% shrinkage). The mean area value of the circle-shaped STSGs was 12.4 $\mathrm{cm}^{2}$ and the median was $12.6 \mathrm{~cm}^{2}$. In the circle-shaped STSGs group, the mean percentage of graft shrinkage was $1.1 \%$ and the median was $0 \%$ [Table 2]. The square-shaped specimens showed primary contraction ranging from $16.0 \mathrm{~cm}^{2}$ ( $0 \%$ shrinkage) to $15.8 \mathrm{~cm}^{2}$ (1.3\% shrinkage). The mean and median area values of the square-shaped STSGs were both $16.0 \mathrm{~cm}^{2}$. In the square-shaped STSGs group, the mean percentage of graft shrinkage was $0.31 \%$ and the median was $0 \%$ [Table 2]. The different shrinkage rates between square- and circle-shaped STSG specimens were not statistically significant $(P=0.33)$.

\section{DISCUSSION}

Skin graft contraction is a common problem resulting in significant morbidity with restriction of joint mobility and cosmetic complications, often requiring multiple corrective operations. Secondary contraction has received the most research emphasis due to the fact that it is clinically more important than primary contraction. Secondary contraction often results in severe effects on body function or patient appearance. Studies on the cellular activity underlying skin graft contraction support a most probable theory that the contraction occurs secondary to the differentiation of fibroblasts to myofibroblasts with expression of $\alpha$-actin filament bundles which exert an inward pull on the wounds edges. ${ }^{[1,9,10]}$ The myofibroblasts have contractile properties similar to smooth muscle cells and organize their actin cytoskeleton along the lines of greatest skin tension. ${ }^{[1,11]}$ As the myofibroblasts are adherent both to one another and to the fibronectin-rich wound bed, the entire mass of granulation tissue contracts. ${ }^{[1]}$ Keratinocytes may also

Table 1: First part of the study: comparison between contraction of FTSGs and STSGs

\begin{tabular}{|c|c|c|c|c|c|c|}
\hline $\begin{array}{l}\text { Skin } \\
\text { graft } \\
\text { type }\end{array}$ & $\begin{array}{l}\text { Initial area } \\
\text { value }\left(\mathrm{cm}^{2}\right)\end{array}$ & $\begin{array}{l}\text { Mean area value } \\
\text { after primary } \\
\text { contraction }\left(\mathrm{cm}^{2}\right)\end{array}$ & $\begin{array}{l}\text { Mean percentage of } \\
\text { area value of FTSG to } \\
\text { the initial area value (\%) }\end{array}$ & $\begin{array}{c}\text { Mean } \\
\text { percentage of } \\
\text { shrinkage (\%) }\end{array}$ & SD & $\begin{array}{c}P \\
t \text {-test FTSG } \\
\text { versus STSG }\end{array}$ \\
\hline FTSG & 12.56 & 11.5 & 87.96 & 12.04 & 0.069 & 0.0011 \\
\hline STSG & 16 & 14.9 & 93.13 & 6.87 & 0.052 & \\
\hline
\end{tabular}

FTSG: Full thickness skin graft, STSG: Split thickness skin graft, SD: Standard deviation

Table 2: Second part of the study: evaluation of the role of shape of skin graft in primary skin contraction

\begin{tabular}{|c|c|c|c|c|c|c|}
\hline Skin graft type & $\begin{array}{l}\text { Initial area } \\
\quad \text { value } \\
\left(\mathrm{cm}^{2}\right)\end{array}$ & $\begin{array}{l}\text { Mean area value } \\
\text { after primary } \\
\text { contraction }\left(\mathrm{cm}^{2}\right)\end{array}$ & $\begin{array}{l}\text { Mean percentage of } \\
\text { area value of FTSG to } \\
\text { the initial area value (\%) }\end{array}$ & $\begin{array}{c}\text { Mean } \\
\text { percentage of } \\
\text { shrinkage (\%) }\end{array}$ & SD & $\begin{array}{c}P \\
t \text {-test circle } \\
\text { versus square }\end{array}$ \\
\hline \multicolumn{7}{|l|}{ FTSG } \\
\hline Circle shaped FTSGs & 12.56 & 11.5 & 87.96 & 12.04 & 0.069 & 0.0011 \\
\hline Square shaped FTSGs & 16 & 14.9 & 93.13 & 6.87 & 0.052 & \\
\hline \multicolumn{7}{|l|}{ STSG } \\
\hline Circle shaped STSGs & 12.56 & 11.83 & 94.17 & 5.83 & 0.021 & 0.142 \\
\hline Square shaped STSGs & 16 & 15.34 & 95.85 & 4.15 & 0.016 & \\
\hline
\end{tabular}

FTSG: Full-thickness skin graft, STSG: Split-thickness skin graft, SD: Standard deviation 
play a distinct role at the early stages of contraction, since studies have shown that keratinocytes are capable of inducing collagen gel contraction in vitro. ${ }^{[12-15]}$ The actinfilament organization within keratinocytes at the wound margin appears to be responsible for the epidermal "purse-string phenomenon". ${ }^{[14]}$ In addition, cytokines and growth factors such as ransforming growth factor- $\beta 1$, insulin-like growth factor and fibroblast growth factors have also been found to play a major role in secondary contraction. ${ }^{[15]}$ Unlike secondary contracture, which is the result of a prolonged biological process, primary skin graft contraction is mainly an immediate physical change in graft dimensions mediated by the tough fibrous layer of the dermis, which is primarily composed of collagens, glycosaminoglycans and elastins.

Davis and Kitlowski ${ }^{[3]}$ were the first to study the primary contraction of skin grafts. The authors used skin grafts from patients of various age and donor sites and recorded the percentage of skin contracture in relation to the thickness of the graft. Their results showed that, regarding the "whole thickness skin grafts" (FTSGs), the mean amount of shrinkage was $43.6 \%$ with little variations according to the donor site. The "half thickness skin grafts" (mid thickness split thickness STSGs) were presented with a mean shrinkage of $24.86 \%$ and the "thick Ollier-Thiersch grafts" (small grafts with thinner periphery and thicker-centered STSGs) with a mean shrinkage of $11.26 \%$ and $11.95 \%$ for abdominal and thigh donor sites, respectively. The very thin "true Ollier-Thiersch grafts" (thin thickness STSGs) demonstrated a greatly reduced primary contraction of $1.24 \% .{ }^{[3]}$ According to the authors, the shrinkage observed was in direct relation to the amount of dermis included in the harvested skin grafts. Using specimens from humans, however, had the limitation that the grafts and their donor sites could not be standardized according to the site, size and shape of the examined grafts. Homogeneity of the samples was further compromised due to variables like gender and age of the studied subjects.

Other authors had previously referred to the etiology of skin graft contraction, coming to the conclusion that the network of elastic fibers of the dermis is responsible for its ability to stretch under the movement of the underlying tissues, as well as for the shrinkage of the skin graft. ${ }^{[16]}$ In Ragnell's study on the secondary contracting tendency of free skin grafts, the elasticity of circular pieces of rabbit skin was estimated using a manometer device. The author concluded that rabbit skin presented uniform elasticity, but no further studies on primary skin graft contraction were performed. ${ }^{[16]}$

Skin is a very complex, integrated, dynamic organ that has many functions. In mammals, the primary functions of the skin include insulation and temperature regulation, although the role of the skin as an endocrine organ and a critical component of the immune system cannot be ignored. ${ }^{[17]}$ Species differences in all of these functions may dramatically alter skin behavior regarding its mechanical characteristics or drug absorption. When barrier, pelage, vascular, endocrine and immunological properties are considered en masse, pigskin is very similar to human skin. ${ }^{17]}$ Pigskin resembles human skin in both structure and function, having similar sparse hair coating, a relatively thick epidermis, similar turnover kinetics, lipid composition, carbohydrate biochemistry, lipid biophysical properties, and - what is most relevant to the present study - a similar arrangement of dermal collagen and elastic fibers. ${ }^{[9,18]}$ All these similarities establish the pig to be an essential model in cutaneous research. Since in vivo experiments on primary skin grafts would require grafts of different shapes, minimum dimensions of 2 or $3 \mathrm{~cm}$, symmetrical locations of the grafts and suitable controls, human experimental material is not available.

In our study, the skin grafts were harvested from pigs, resulting in standardized specimens in terms of size, shape and location of the donor site and at the same time, skin behavior close to that of human skin. The substantial differences between our results and the results reported by Davis and Kitlowski, ${ }^{[3]}$ however, point out the different primary contraction behavior of human and porcine skin. Although similar in many ways, different thicknesses and possibly different elastic properties between human and porcine skin may lead to different contraction behaviors of skin grafts. Furthermore, the rate of primary skin contraction probably depends on donor site characteristics. Clinical experience shows that skin harvested from the backs of patients presents limited contraction when compared with skin grafts from other sites. Furthermore, there was one specimen from the STSG group of the first part of the study that surprisingly showed $0 \%$ shrinkage. Since $0 \%$ primary skin contraction before is rather unusual, we believe that this behavior was related to a specific donor site and will be further investigated in upcoming studies. Another possible reason for such a discrepancy in skin graft shrinkage could be technical difficulties: harvesting very thin skin grafts with small amounts of dermismay have been responsible for graft contraction.

The mean percentage of primary skin graft contraction has found to be different in the two parts of the study, with mean values for both FTSG and STSG contraction showing inconsistencies between the study series. These unexpected differences were probably due to the small number of specimens in the second part, which did not yield statistically significant results. Furthermore, the use of only one animal in the second part of the study may have magnified the role of biological variation, a matter that will be further investigated in future larger studies.

To the best of our knowledge, primary skin contraction in relation to the shape of the skin graft has never been investigated before. In our study the mean graft shrinkage was $5.8 \%$ and $4.2 \%$ in circle-shaped and square-shaped FTSGs, respectively [Table 2]. The difference was more notable in the STSGs, where the circle-shaped specimens showed graft shrinkage of $1.1 \%$, whereas the square-shaped present a mere $0.31 \%$ [Table 2]. The difference recorded could be due to the relation of the line of contraction with the skin tension lines. Square specimens have one contraction vector that runs parallel to the direction of the skin tension lines, whereas the round-shaped specimens have multiple contraction 
vectors positioned at various angles to the skin tension lines. Theoretically, the projection of the skin tension lines to the radii of the circle-shaped specimens could possibly add to their total graft shrinkage. Due to the small number of specimens, however, further studies should be conducted in order to determine potential statistically significant findings.

Mean percentage of primary contraction for square STSGs was found to be 3.8 times higher than round STSGs ( $4.2 \%$ and $11 \%$ for square and circular STSGs, respectively). The recorded difference for the FTSGs, however, is not that prodigious. Since this is only a preliminary report with a small number of specimens involved, we believe that future studies will help to clarify the issue.

Limitations regarding human specimens necessitate the use of animal models; further studies are required in order to investigate whether pigskin is suitable for the study of primary graft contraction. The study cohort is limited and a larger series for all arms is needed for a better understanding of these phenomena.

Skin grafts are widely used and any information regarding their characteristics is valuable. Our preliminary report reveals an expected increased shrinkage of FTSGs compared to STSGs and in a limited number of specimens, the shape of the skin graft seems to affect primary contraction of the STSGs. Although it is difficult to dramatically change the shape of skin grafts, if this feature is ultimately found to alter primary contraction, the results could possibly be applied in clinical practice.

\section{REFERENCES}

I. Harrison CA, MacNeil S. The mechanism of skin graft contraction: an update on current research and potential future therapies. Burns 2008;34:153-63.

2. Walden JL, Garcia H, Hawkins H, Crouchet JR, Traber L, Gore DC. Both dermal matrix and epidermis contribute to an inhibition of wound contraction. Ann Plast Surg 2000;45: 162-6.
3. Davis J, Kitlowski E. The immediate contraction of cutaneous grafts and its cause. Arch Surg 1931;23:954-65.

4. Hinshaw JR, Miller ER. Histology of healing split-thickness, full-thickness autogenous skin grafts and donor sites. Arch Surg 1965;91:658-70.

5. Corps BV. The effect of graft thickness, donor site and graft bed on graft shrinkage in the hooded rat. Br J Plast Surg 1969;22:125-33.

6. Jones T, McDonald S, Deitch EA. Effect of graft bed on long-term functional results of extremity skin grafts. J Burn Care Rehabil 1988;9:72-4.

7. Davies DM. Plastic and reconstructive surgery. Scars, hypertrophic scars, and keloids. Br Med J (Clin Res Ed) 1985;290: 1056-8.

8. Kilkenny C, Browne W, Cuthill IC, Emerson M, Altman DG, NC3Rs Reporting Guidelines Working Group. Animal research: reporting in vivo experiments: the ARRIVE guidelines. BrJ Pharmacol 2010;160:1577-9.

9. Tomasek JJ, Haaksma CJ. Fibronectin filaments and actin microfilaments are organized into a fibronexus in Dupuytren's diseased tissue. Anat Rec 1991;230:175-82.

10. Welch MP, Odland GF, Clark RA. Temporal relationships of F-actin bundle formation, collagen and fibronectin matrix assembly, and fibronectin receptor expression to wound contraction. J Cell Biol 1990; I 10:133-45.

II. Petroll WM, Cavanagh HD, Barry P, Andrews P, Jester JV. Quantitative analysis of stress fiber orientation during corneal wound contraction. J Cell Sci 1993; 104:353-63.

12. Denefle JP, Lechaire JP, Zhu QL. Cultured epidermis influences the fibril organization of purified type I collagen gels. Tissue Cell 1987;19:469-78.

13. Souren JM, Ponec M, van Wijk R. Contraction of collagen by human fibroblasts and keratinocytes. In Vitro Cell Dev Biol 1989;25:1039-45.

14. Brock J, Midwinter K, Lewis J, Martin P. Healing of incisional wounds in the embryonic chick wing bud: characterization of the actin purse-string and demonstration of a requirement for Rho activation. J Cell Biol 1996; I 35: 1097-107.

15. Wang YB, Ogawa Y, Kakudo N, Kusumoto K. Survival and wound contraction of full-thickness skin grafts are associated with the degree of tissue edema of the graft bed in immediate excision and early wound excision and grafting in a rabbit model. J Burn Care Res 2007;28:182-6.

16. Ragnell $A$. The secondary contracting tendency of free skin grafts; an experimental investigation on animals. Br J Plast Surg 1952;5:6-24.

17. Monteiro-Riviere NA, Riviere JE. The pig as a model for human skin research. In: Swindle M, Bouchard GF, editors. Swine in Biomedical Research: Update on Animal Models. Auxvasse: Sinclair Research Center; 2005. p. 17-22.

18. Monteiro-Riviere N. Comparative anatomy, physiology, and biochemistry of mammalian skin. Boca Raton: CRC Press; 199I. p. 3-7I.

How to cite this article: Berezovsky AB, Pagkalos VA, Silberstein E, Shoham Y, Rosenberg L, Krieger Y. Primary contraction of skin grafts: a porcine preliminary study. Plast Aesthet Res 2015;2:22-6.

Source of Support: Nil, Conflict of Interest: None declared.

Received: 24-04-2014; Accepted: 28-10-2014 